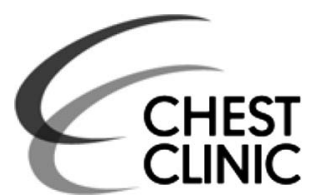

${ }^{1}$ Oxford Centre for Respiratory Medicine, Oxford University Hospitals NHS Foundation

Trust, Oxford, UK

${ }^{2}$ University of Oxford Respiratory Trials Unit, Churchill Hospital, Oxford, UK ${ }^{3}$ Department of Radiology, Oxford University Hospitals NHS Foundation Trust,

Oxford, UK

${ }^{4}$ NIHR Oxford Biomedical Research Centre, University of Oxford, Oxford, UK

\section{Correspondence to}

Dr John P Corcoran, Oxford

Centre for Respiratory

Medicine, Churchill Hospital,

Oxford OX3 7LE, UK;

jpcorcoran@doctors.org.uk

Received 11 July 2016 Revised 22 August 2016 Accepted 2 September 2016 Published Online First 28 September 2016

\title{
Large airways nodularity secondary to tracheobronchopathia osteochondroplastica
}

\author{
John P Corcoran, ${ }^{1,2}$ John M Wrightson, ${ }^{1}$ Alastair J Moore, ${ }^{1}$ Fergus V Gleeson, ${ }^{3,4}$ \\ Annemarie Sykes ${ }^{1}$
}

A 74-year-old retired librarian was referred for assessment after a full-body CT, performed to investigate a 3-month history of persistent nausea and weight loss, incidentally identified a $10 \mathrm{~mm}$ left lower lobe nodule alongside small volume nodularity seen throughout the trachea and proximal main bronchi (figure 1). There was no previous thoracic imaging for comparison. She had a five pack-year smoking history and reported no preceding respiratory disease or symptoms. Her medical background was otherwise unremarkable. She appeared well in clinic; physical examination, basic spirometry (FEV1 131\%, FVC 133\% predicted) and flow-volume loops were normal. A subsequent positron emission tomography-CT scan demonstrated physiological uptake only and no areas of abnormality. Following MDT discussion it was recommended to obtain histopathological samples via bronchoscopy given the history of unexplained weight loss and extent of the nodularity within the large airways.

Flexible bronchoscopy demonstrated multiple smooth discrete nodules of varying size throughout the trachea and extending to a lesser extent into the proximal main bronchi (figure 2). These nodules were firm to contact with macroscopically normal overlying mucosa; they were anatomically associated with cartilaginous rings, sparing the posterior membranous wall of the large airways. Multiple endobronchial biopsies and brushings were taken from separate nodules in the distal trachea and proximal right main bronchus without complications. Histology demonstrated normal bronchial mucosa with no evidence of malignancy or other pathological process. On the basis of the clinical presentation and investigation findings a diagnosis of tracheobronchopathia osteochondroplastica (TPO) was made.

TPO is an unusual benign chronic disease characterised by multiple osteocartilaginous submucosal nodules that originate from the cartilaginous rings of the airways, and therefore classically spare the posterior membranous wall. ${ }^{1} 2$ This unique geographic distribution helps differentiate TPO from other conditions that might also cause nodularity of the large airways such as neoplastic disease, endobronchial sarcoid, TB and amyloidosis. The macroscopic appearance of the nodules, being clearly associated with cartilaginous rings and typically having normal overlying endobronchial mucosa; and their hardness that can make biopsies difficult to obtain are additionally reassuring when differentiating TPO from malignant disease. The aetiology of the condition is unknown with postulated theories including infective, traumatic and environmental stimuli (notably silica). The nodules in TPO are typically seen in the distal two-thirds of the trachea and proximal main bronchi, although they can involve any part of the bronchial tree. The true incidence of TPO in the general population is uncertain; with postmortem, bronchoscopic and radiological studies quoting figures of anywhere between $1: 200$ and $1: 1000 .^{1}{ }^{3}$ It does, however, certainly go unrecognised in many cases due to a lack of awareness among clinicians.

Patients may present with non-specific symptoms including chronic cough, haemoptysis, wheeze and dyspnoea; although many cases are asymptomatic and picked up incidentally during investigations for an unrelated condition. While imaging studies, notably CT, can be suggestive of TPO, direct visualisation at bronchoscopy remains the definitive test
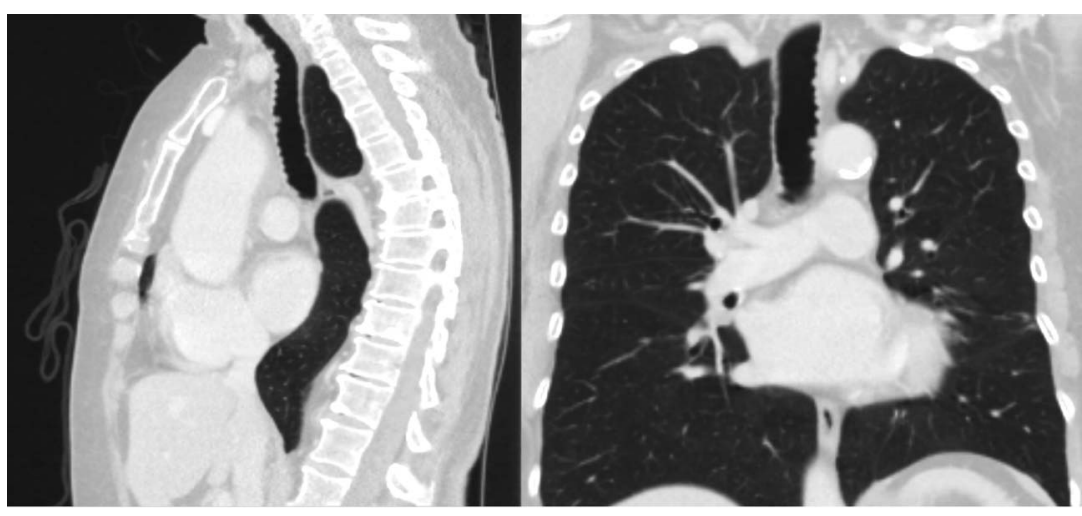

Figure $1 \mathrm{CT}$ images demonstrating irregular nodularity throughout the trachea with calcification and sparing of the posterior wall. 


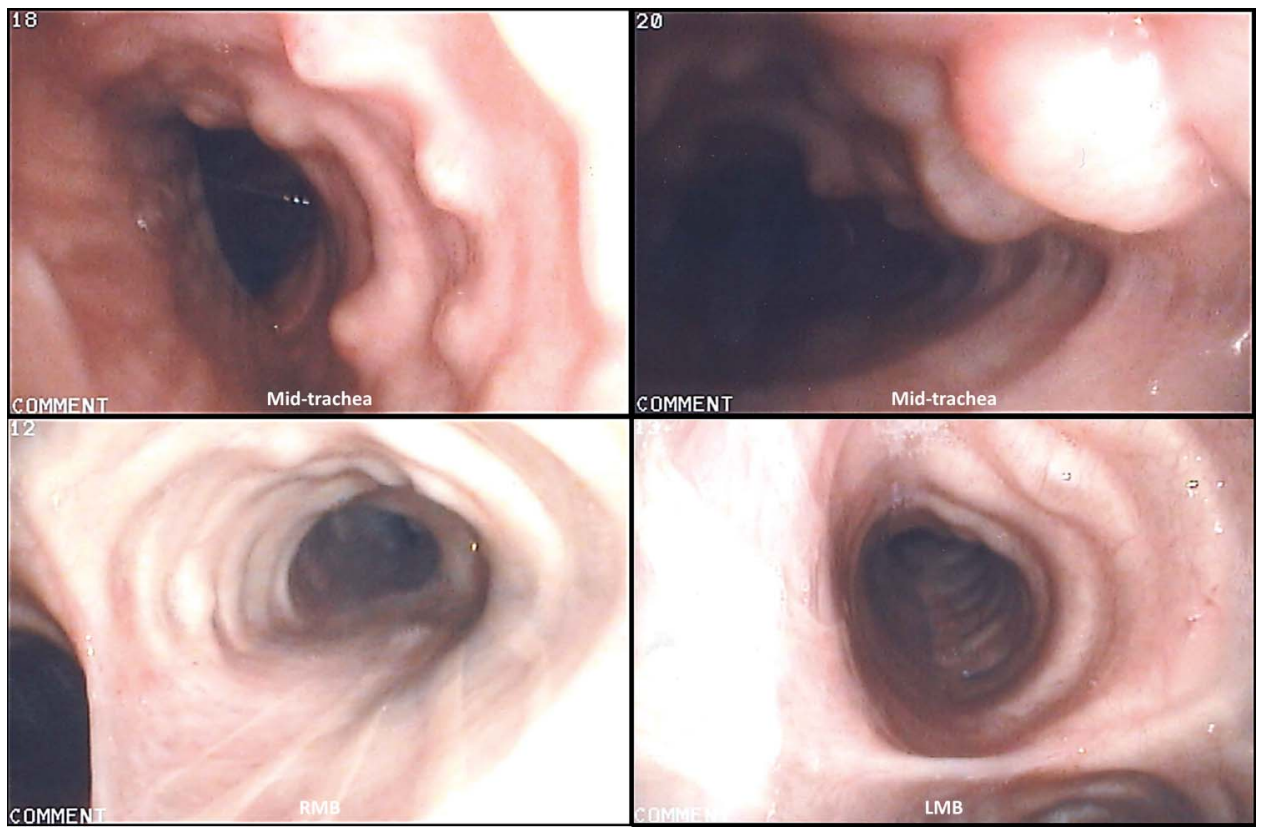

Figure 2 Bronchoscopic images demonstrating multiple discrete smooth nodules distributed along the anterolateral airway walls in association with cartilaginous rings, macroscopically diagnostic of tracheobronchopathia osteochondroplastica. LMB, left main bronchus; RMB, right main bronchus.

with the macroscopic appearance alone usually sufficient to make a diagnosis. ${ }^{1} 2$ TPO is generally regarded as a benign condition, but can be complicated by recurrent respiratory infections and progressive airways obstruction as the lesions impair both airflow and ciliary clearance. Treatment is limited to these symptomatic patients and varies from conservative medical measures, such as bronchodilators and antibiotics, to endobronchial intervention for more severe cases with extensive airway involvement and associated obstruction. ${ }^{12}$ In the absence of any respiratory symptoms or prior imaging for comparison, our patient is being managed conservatively with a plan for 1-year interval imaging to monitor her parenchymal pulmonary nodule. Her nausea and weight loss resolved spontaneously without a clear cause being identified.
Contributors Article conception: JPC, AS. Initial manuscript preparation: JPC. Preparation of images: JPC, FVG, AS. Final manuscript appraisal and revision: JMW, AJM, FVG, AS. All authors were involved in the clinical management of the patient.

Funding FVG is supported by the NIHR Oxford Biomedical Research Centre.

Competing interests None declared.

Patient consent Obtained.

Provenance and peer review Not commissioned; externally peer reviewed.

\section{REFERENCES}

1 Prakash UB. Tracheobronchopathia osteochondroplastica. Semin Respir Crit Care Med 2002;23:167-75

2 Chroneou A, Zias N, Gonzalez AV, et al. Tracheobronchopathia osteochondroplastica. An underrecognized entity? Monaldi Arch Chest Dis 2008;69:65-9.

3 Hussain K, Gilbert S. Tracheopathia osteochondroplastica. Clin Med Res 2003:1:239-42. 